\title{
Smart Company System using Hybrid Nomenclature of Neural Network
}

\author{
Mbida Mohamed $^{1}$, Ezzati Abdellah ${ }^{2}$ \\ Department of Emerging Technologies Laboratory (LAVETE) \\ Faculty of Sciences and Technology Hassan 1st University \\ Settat, Morocco
}

\begin{abstract}
Physically, to manage the data related to the products, CRM, suppliers and Administration warehouse of the company makes us use a lot of human resources, and a time which deals with this, consequently the error rate increases and sometimes everything goes out of control, however, this work designed an intelligent overall management system (an intelligent neural network) which completes and up-date the product management network that presented in one of the previous articles. This new version assembles the three modules, in an order to automate tasks in the real time.
\end{abstract}

Keywords-Neural; network; intelligent; CRM; company; warehouse; real time

\section{INTRODUCTION}

\section{A. Standard Logistics Inventory Administration}

These days, when talking about stock administration, it's truly about keeping and following an amount of merchandise in a store. It is an action that separates into: the board of developments passage and leaves products; recharging the executives; lastly a related assignment the administration of the article documents. Contingent upon the association of the organization, these errands can be doled out to an individual or two diverse staff profiles.

The vendor for the execution of the physical developments of passage and exit;

- Supply Management for stock following and recharging

- Agent gathering data about every item by a gadget standardized tag committed to peruse the bare-code scanner.

Notwithstanding, most industry divisions use standardized tags from numerous points of a view.

Standardized identifications have altered the creation, preparing and observing of items in the food, bundling, retail, clinical, a drug, gadgets, a car, mechanical segments and air transportation.

Bare code Scanner can be found on all electronic and mass-market items, from the battery of your cell phone to the case containing your new sport shoes. The utilization of 1-D and 2-D codes decreases overhead expenses via mechanizing and disentangling a gracefully chain the executives, stock, choice, and the buying cycle. The modern segment has additionally embraced standardized identifications for reasons of the security and duty.

Lately, various nations have started to require makers of clinical and drug items to put precisely comprehensible codes on all bundling, including singular medication bundles. On the off chance that a flawed item is conveyed to a drug store, the programmed following of each bundle will quicken the review of items while guaranteeing the accessibility of the value control information all through the flexibly chain.

\section{B. Basic Customer Relationship Management}

Most companies use macro dashboards to manage customer data, which can be tedious to enter and update when there are many customers, or they are using phone calls to prospect the customer's opinion in order to develop the quality of service, this can generate errors which can be costly to the company, which implies to find a solution to automate in realtime all these transactions with the customers without fail.

\section{Standard Supplier Service}

Each company that manufactures a finished product, needs raw or semi-finished materials, so a continuous relationship with suppliers is required by email or a phone, to have traceability it needs to save invoice information suppliers, then an intelligent and flexible solution is necessary so as not to weigh down the task for a person who will do this again a hundred or more times.

\section{Classical Neural Networks}

By relationship with organic neurons a network of artificial neurons must have the option to learn and recreate "smart" thoughts in an artificial mechanism (every interneuronal network connection will have the option to adjust and develop as learning advances).

The proper neuron is a model that is portrayed by an inside state $\mathrm{s} \in \mathrm{S}$, input signals $\mathcal{X} 1 \cdots \mathcal{X}_{\mathrm{p}}$ and the activation function (Formula 1)

$\boldsymbol{s}=h\left(\chi_{1} \cdots \chi_{2}\right)=g\left(\alpha_{0}+\chi_{\mathrm{j}}\right)=g\left(\alpha_{0}+\alpha^{\prime} \chi\right)$

The activation function [1] plays out a change of a relative mix input signals, $\alpha 0$, steady term, being known as the predisposition of the neuron. This relative mix is dictated by a weight vector.

$\left[\begin{array}{llll}-\alpha & 0 \ldots & \alpha & p\end{array}\right]$ related with every neuron and whose qualities are assessed in the learning stage. 
They comprise the memory or disseminated information on the network. The various versions of neurons are recognized by the idea of their activation function. The principal types are:

- Linear $\mathrm{g}$ is the identity function,

- Threshold $g(\mathcal{X})=1[0 ;+\infty[(\mathcal{X})$,

- $\operatorname{Sigmoid} g(\mathcal{X})=1 /(1+\mathrm{e} X)$,

- $\operatorname{ReLU} \boldsymbol{g}(\mathcal{X})=\max (0, \mathcal{X})$ (rectified linear unit),

- $\quad$ Radial $g(X)=\sqrt{ }(1 / 2) \pi$ e $(-X 2 / 2)$,

- Stochastic $g(\mathcal{X})=1$ with probability $1 /(1+\mathrm{e}-\mathcal{X} / \mathrm{H})$,

- otherwise 0 (H acts as a temperature in a simulated

- $\quad$ annealing Pseudo Code).

This article, describe a hybrid intelligent architecture made-up that joins neuron networks as a learning system on Item Data, CRM and Suppliers interaction, in order to automate the general system of the company.

\section{RELATED WORKS}

Neural Network (NN) innovation has been effectively applied in numerous business territories, and a few works have been done, we quote the most significant

\section{A. Estimating Model of Supply Chain Administration based on Neural Network}

Pr HongJing Liu continue the utilizations of neural organization innovation [2] in gracefully chain the executives, which contain three spaces: streamlining, estimating and choice help. Nonetheless, the Back Propagation (BP) the NN is applied to gauge the interest of the bicycle in a definite area, the estimating result show that BP neural network has more noteworthy guaging exactness than that of customary determining model.

\section{B. Artificial Neural Network for Transportation Infrastructure Systems}

Artificial Neural Networks (ANNs) depict as the general interconnection of the frameworks along with numeric weighting that can be tuned dependent on experience, framework Inputs, Processing and Outputs. Additionally, the genuine bit of leeway of ANNs is the capacity to clarify complex framework issues, for example, one which are found inside the Transportation Infrastructure System's [2]. ANNs for Transportation Infrastructure System must consolidate framework designing strategies that will be economical for future years and kept up at satisfactory levels. Appropriately, Though Pr Koorosh Gharehbaghi present the idea of ANNs and its center capacities for the advancement of Transportation Infrastructure Systems specifically the support measures.

\section{Predicting Logistics Delivery Demand with Deep Neural Networks}

Conveyance time impacts the logistics route coordination's, contingent upon the necessities of the place and quantity. An effective forecast of conveyance request would help the organization of logistics model. The data on conveyance request are time-reliance and space-relationship. Displaying the multidimensional grouping or building the expectation dependent on it would be a calculation expending work. Anyway this examination depends on profound figuring out how to propose an effective strategy to foresee conveyance request. With the reenactment study, the expectation performance $[3,4]$ of the proposed technique is satisfactory. This is helpful for the further investigation of coordinations logistic choices making.

\section{Social CRM and Suppliers using Web Mining}

Conventional CRM (Customer RelationshipThe board) contains three modules, Marketing, Sales, and Backing, which depend on the client relationship and profiling data. While the data contained in those three modules is contribution by administrator, N. Karna and others [5] accumulate considerably more data from the Internet. can discover connection between clients and discover their profile from the Internet. This data can be utilized to improve and coordinate the CRM to perform better in supporting the business destinations. Social event data from the Internet implies that need Information Recovery and Information Extraction that include numerous sources from Internet, for example, webbased media, net blog, and news. This research gives the model of information mining usage in customary CRM to become social CRM. This exploration contributes for CRM upgrade where client driven application gets robotized.

\section{SMART COMPANy CONTROL SySTEM BASED ON HYBRID NN NOMENCLATURE}

Each company has three essential modules (CRM, Suppliers, Administration inventory) to properly manage the manufacturing processes, however this work implement an intelligent system that automates tasks without human intervention by a neural network that saves and continuously learns, that will be detailed later in this article, which will follow module by module (Fig. 1).

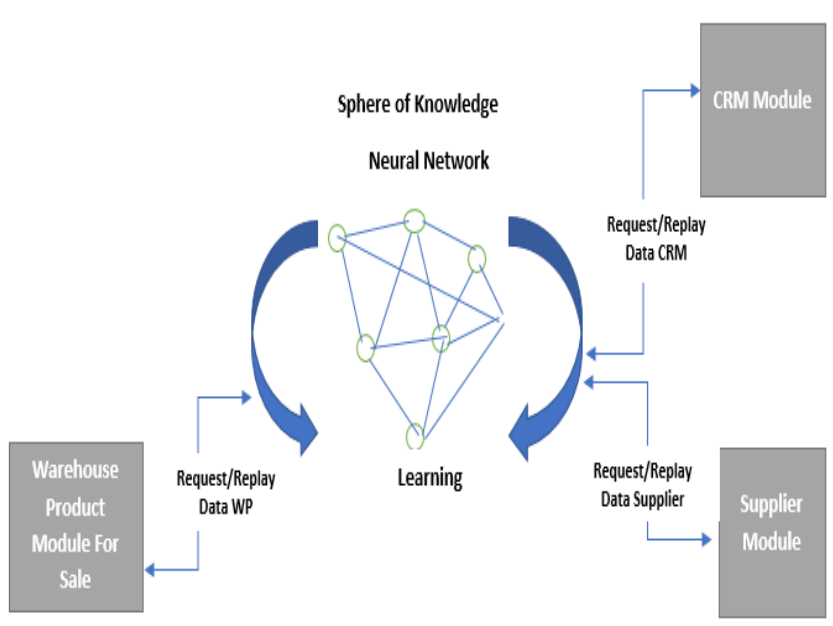

Fig. 1. Smart System Company in Interaction with the Four Modules. 


\section{A. Smart Administration Warehouse of Product Module for} Sale

1) Nearest Neighbor data classic inventory administration: Nowadays Tendency is the mobility, if the data capacity is away from the PC, and the items are profound, it can't carry the items to the PC: it is important to works distantly. Some might want to deal with their stock with a phone, yet they are not "made for". There are additionally tablets or PDAs, however practically speaking you have your hands involved. That is the reason they utilize moderately basic bare-code readers, whose solitary capacity is to peruse standardized identifications and enter amounts. These readers are more powerful contrasted with tablets or PDAs, and particularly more affordable (Fig. 2).

The work is done in two phases:

- Scan items and quantities in shelves;

- Loads information saved on the PC.

This method of activity called "cluster" is sheltered and dodges dull control on a little screen. Anyway the filtering of the items just as the taking care of the data on $\mathrm{PC}$, requires strategic operators to do these undertakings which can create errors during the assortment or stacking of the information (Forgets of checking/capture of the items ...).In expansion another detriment can be in the loss of time in the two stages to have an information base took care of, on account of an update of the capacity stores need to experience similar advances, which produces some other time lost. During the connectivity of the hand shower with the PC, there might be network issues (driver, connector, coding...) Or the inside memory of the code bar reader might be depleted whenever since its stockpiling is restricted to $8 \mathrm{MB}$. For this the current work has built up an insightful stock administration system to lessen the pace of mistakes that can happen in this cycle of information extraction and obtaining, and furthermore to have ongoing information accessibility.

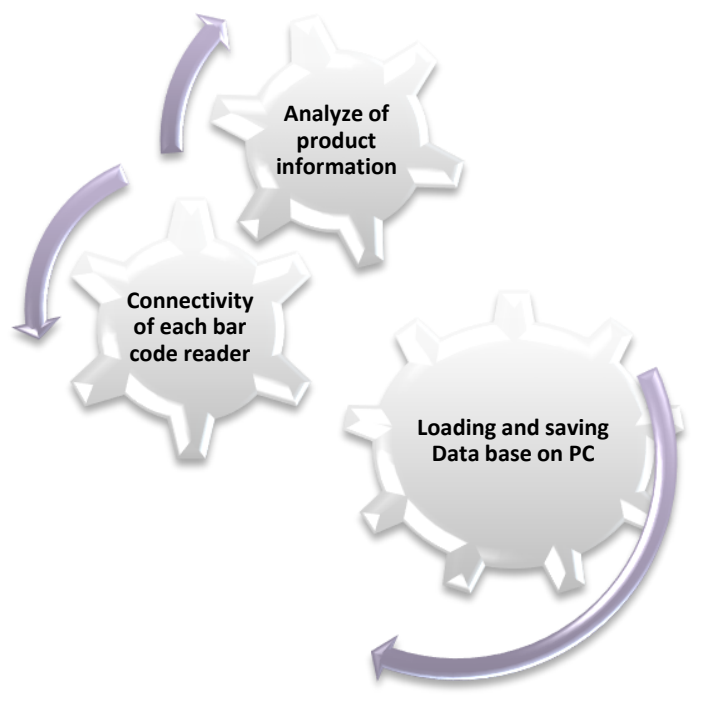

Fig. 2. Classical Inventory Administration.
2) Hybrid nemenclature implementation for warehouse product module for sale: Today, the traditional stock administration causes many losses of time and human resources just as issues with information securing. To advance this administration cycle, this requires the improvement of an incredible powerful hybrid framework dependent on Cognex technology of vision 1.2-dimensional scanner's, with sensors set up in the distribution center and an intelligent neural network with a bar-code identification, which permits the reinforcement and learning of an information in these circles of data. The execution of this design requires a section through three stages introduced in the clear figure above (Fig. 3), in what follows this article will clarify each part and its fucntion [5].

3) Collection of information produced by $1 D / 2 D$ vision technology sensors: When gathering item informations, this task need the vision sensors technology, which are such an advanced camera set before each item that snaps a photo of the code. A microprocessor running an special picture preparing programming distinguishes and interprets the code before moving the acquired information to the neural netwrok (Fig. 4) [5]. One of the fundamental elements of decision of a picture sensor, or camera, is its goal picture, in other words the quantity of pixels that make up every photo.

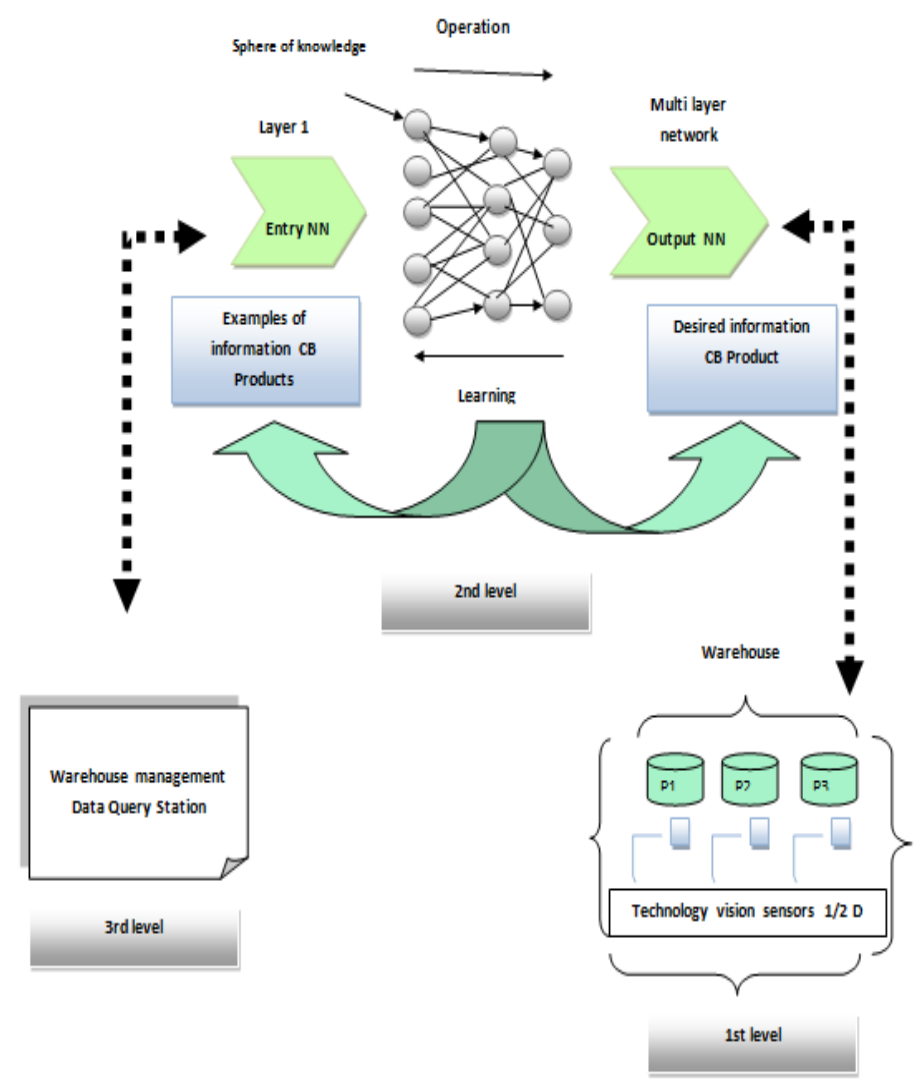

Fig. 3. Hybrid Process for Smart Inventory Product Administration for Sale. 


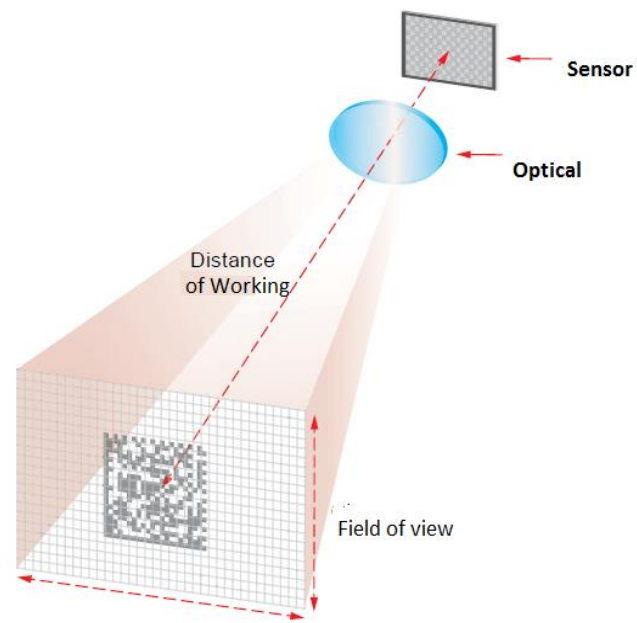

Fig. 4. Pixel Grid of the PPM Value on a Data-Matrix Code using a Vision Technology Reader.

According to a vision technology reader, the pixels resolution [6] per module (PPM). PPM is the quantity of pixels comparing to a phone or module code and ensures the camera has enough resolution to peruse this code. This worth is determined by separating the resolution of the camera one way (for instance, 752 pixels for a standard resolution readers) by the quantity of code modules, at that point doing likewise count the other way, before to increase the two numbers got. This may appear to be convoluted, yet the design applications or picture preparing programming of the mechanical vision technology code readers can rapidly compute the PPM value (Four in the model above).In expansion, new microprocessors and CMOS computerized sensors additionally empower vision-based readers to be close to as quick as the best laser scanners. These advances are notwithstanding the conventional advantages of vision-based drives: no moving parts, longer lasers, capacity to peruse damged or omnidirectional codes, and the capacity to record pictures for review and following purposes or then again to screen code stamping frameworks[7].

Nonetheless, after the gathering of the item data as indicated by the EAN 13 Standard [8], the information is sent back to the neuron network with Wifi, since this vision innovation offers a scope of mechanical correspondence conventions including Ethernet, USB, RS-232, advanced I/O, Ethernet/IP, PROFINET and Modbus TCP/IP.

4) Getting and learning data by $N N$ of administration inventory product module for sale: After the period of data extraction by vision technology sensors, they send these information to the neuron network that was planned with the Java langage, to begin gathering and learning out about every item and remember it, in case if it exists in the underlying data base (.dat). The $\mathrm{NN}$ has been planned in Java language that permits to know and devise the scanner bar-code picture $(1 \mathrm{D} / 2 \mathrm{D})$ as a progression of digits in four sections, so as to perceive each part as indicated by the contry, this manufacturer of this one, item producer and the control key that permits to approve the bare code. Anyway, learning process product data in the accompanying Java code Part (Pseudo Code 1):

\section{Pseudo Code 1: Getting and learning process for NN}

Input:

Int $\mathbf{N}$ : Number of sensors of technology vision barre code List of series bar code received from RCSF: LSCBWn List of series bar code received from fichier des exemples ( ExRN.dat): LSCBF

Output: Informations about products

For each Lwsni $\epsilon$ LSCBWn[N] do

If the Country of Lwsni $>\mathbf{3 0 0} \& \&$ the Country of Lwsni $<379$

Sendto station (" the product is from France ") ;End;

$$
\text { ! }
$$

Lwsni $\leftarrow$ Lwsni+1;end;

For each Lwsni $€$ LSCBWsn[N] do

For each LFi $\boldsymbol{\epsilon}$ LSBF do

If the manufacturing contry of ( Lwsni) $=$ the manufacturing contry of (LFi)

Sendto station (the manufacturing contry of ( Lwsni));

Lwsni $\leftarrow$ Lwsnit+1;

else LFi $\leftarrow$ LFi+1;

else Save into file of exemples (the manufacturing contry of ( Lwsni));

Lwsni $\leftarrow$ Lwsni+1; end;

For each Lwsni $\boldsymbol{\epsilon}$ LSCBWsn[N] do

For each LFi $C$ LSBF do

If the Product of manufacturing of $(\mathrm{Lwsni})=$ the Product of manufacturing (LFi)

Sendto station (the Product of manufacturing of ( Lwsni)) ; Lwsni $\leftarrow$ Lwsnit+1; else LFi $\leftarrow$ LFi+1;

else Save into file of exemples (Product of manufacturing of (

Save into file of exemples (Produc

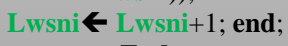
End;

if $(($ series cb[i].ProductFab $)==(($ seriescbF () .ProductFab $))$

\{

System.our.println("The manufactured product is $\mathrm{xxxx}$ ");

if ((series cb[i].Validkey) $==(($ seriescbF ()$\cdot$ validkey $))$

System.our.println("the key is valid ");

End;

\section{B. Smart CRM Module}

According to the general literature, CRM or client relationship management is a methodology for dealing with the connections and cooperation of an organization with its clients or expected clients, which requires flexibility and reduced data processing time to guarantee customer satisfaction, for this reason a CRM module has been designed which is based on an intelligent neural network by learning the customer character and offering him the appropriate product services at its choice, with an inspection of the comments ,and remarks delivered by the latter to ensure a continuous and real-time improvement of the products. 


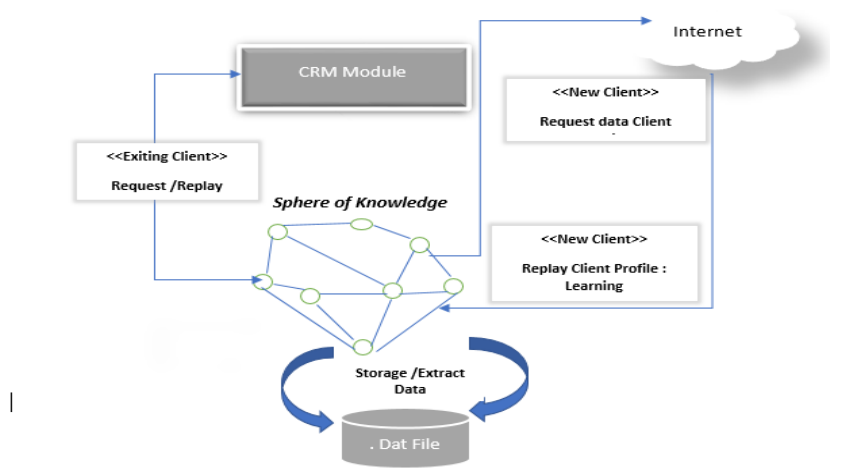

Fig. 5. CRM Module Process Managed by the Smart Company System NN.

\begin{tabular}{|l|}
\hline \multicolumn{1}{|c|}{ Pseudo Code 2: Getting and learning Smart CRM } \\
process \\
Input: \\
Int L number of Exiting Client \\
Exiting Client profile: ECP \\
List of Product Client Profile: LPCP \\
New Client Profile Choices product: NCP \\
Neural Network: NN \\
Output: Informations about Client profile products \\
choice ICP \\
Request ECPx \\
For each ECPi 6 ECP [L] do \\
If ECPi =ECPx \\
Listing to Client LPCP \\
Else \\
Collecting and analyzing NCP From Internet \\
Saving Data NCP in (.dat) File \\
Endif; End ; \\
\hline
\end{tabular}

According to the descriptive figure (Fig. 5), the CRM module consults the base of the neural network in case of a customer already registered and displays the choices of these purchases according to his customer character, in the case of a new customer the neural network solicits the internet to collect profile data and analyze its product choice behavior and save it in the database linked to the NN (Pseudo Code 2).

\section{Smart Supplier Module}

Every productive company needs suppliers, and this must leave continuous contact with them, for this reason it was thought to automate the monthly demand for raw material products by sending invoices to these suppliers by email according to a configured period of time, and the human intervene only in the case of an update of quantity or stop of cooperation with the suppliers, this facilitates the task to the company in an organized way, the Pseudo Code 3 and the Fig. 6 below illustrate this procedure.
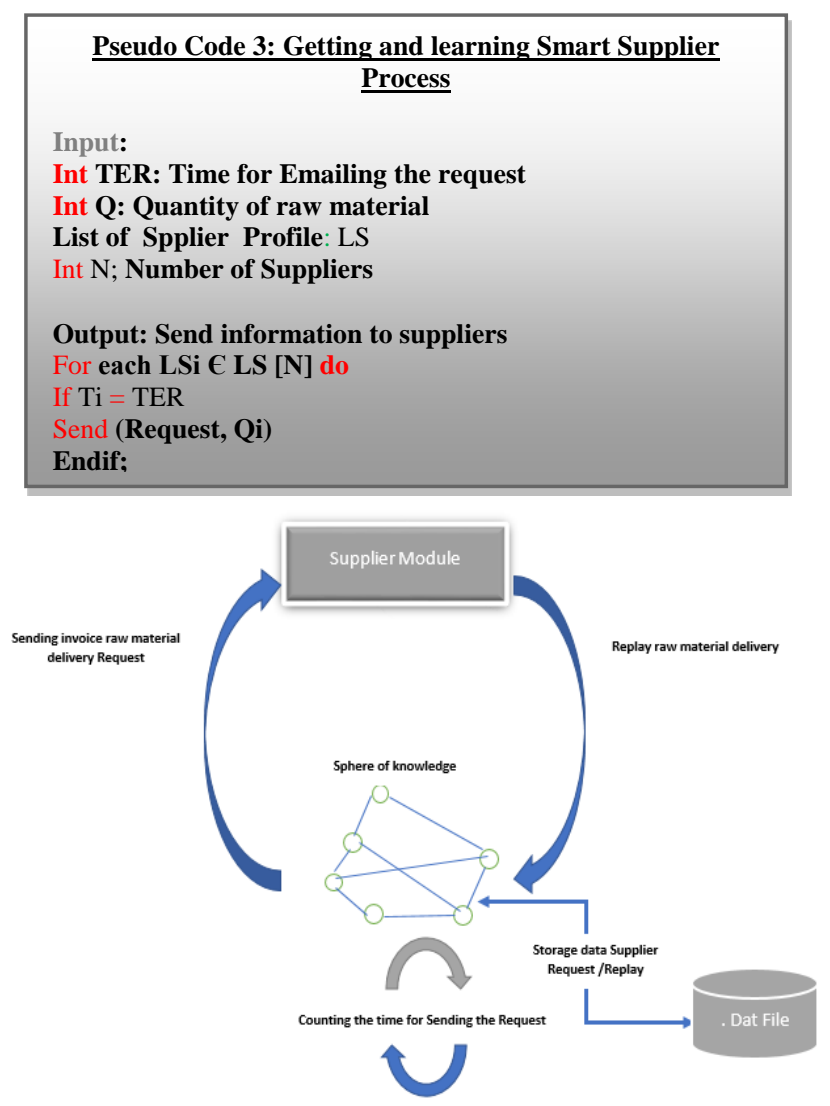

Fig. 6. Smart Supplier Process.

\section{EXPERIMENTS}

From what is above, this work proves that the neural network by learning is the main engine for this intelligent system which includes the three basic modules of the company, with automation and data acquisition in real time, without errors or delay of 'sending. This hybrid architecture dependent converged with a Java model Kohonen [9] has been planned and actualized with neuron and 26 output neurons, For the experimental simulation of the company's three modules, this work will use Any Logic software for scenario modeling and the neural Java network can work interactively with the simulation models, dynamically reading its states and taking action.

NB:

*The models have two validation times: (Validation with customer and the Internal validation) before entering in the production phase.

*The vision sensors technology of inventory administration module are simulated in the Any Logic platform as a 2/3 D bar code information transmitter.

Each module is characterized by an interactive interface linked to the Smart model company (Fig. 7, 8, 9). 


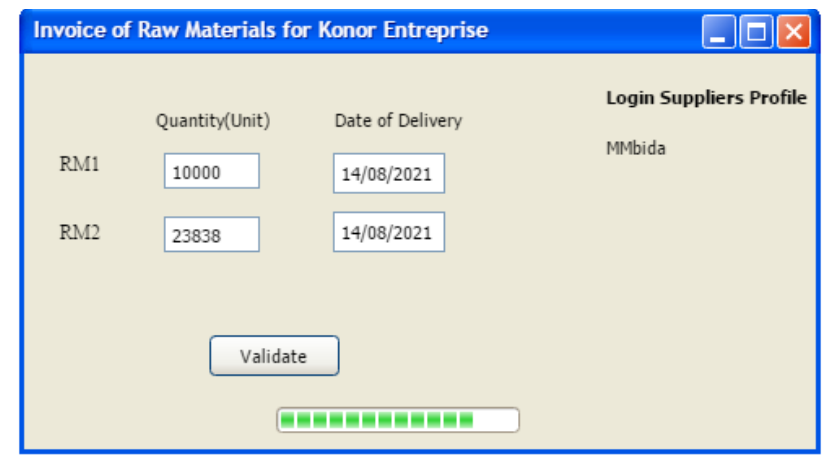

Fig. 7. Automated Invoice for Raw Materials.

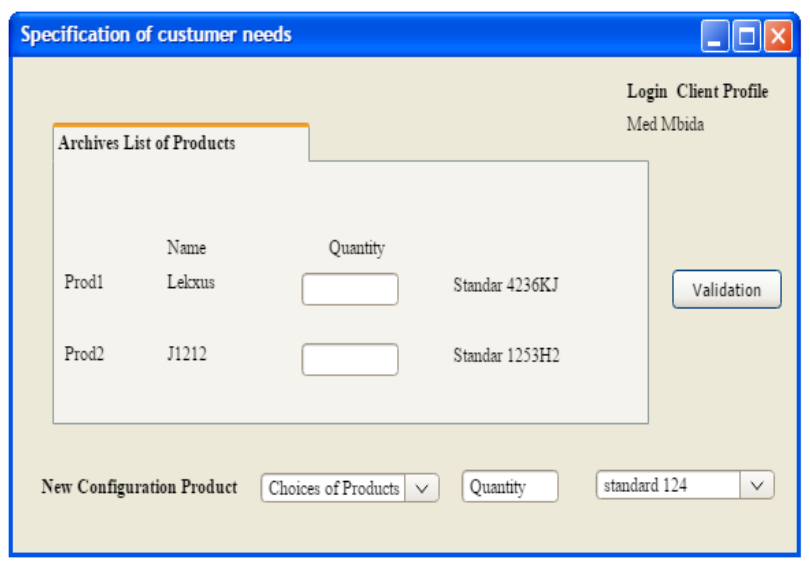

Fig. 8. Specification of Costumer Needs.

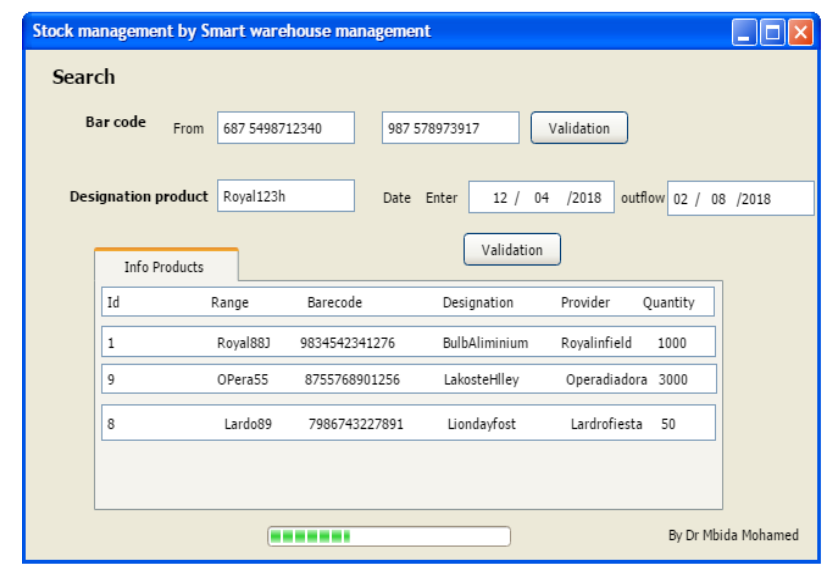

Fig. 9. Smart Administration of Products.

\section{A. Statistics Study}

The simulation of these getting and learning data scenarios was done first on the Encog 3.3 platform to run the intelligent neural network in Java, in 2nd step this work realized a model at the production company, on the Any Logic software, and then we made the connection between the neural network and the business model.

The general simulate company is presented in Main Model according to the manufacture of three products (Fig. 10), and also this work present the models associated with the three modules (Suppliers, CRM, Finished products) (Fig. 11, $12,13)$.

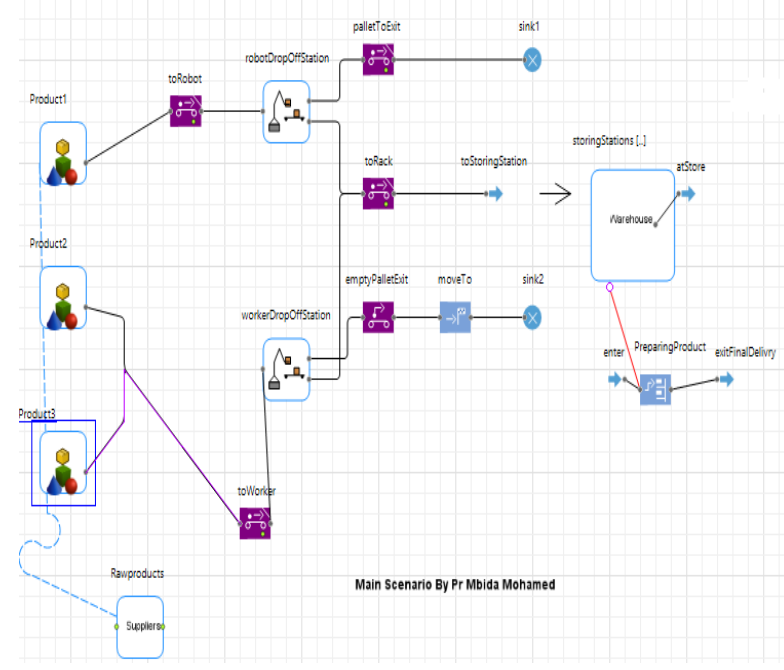

Fig. 10. Main Company Scenario.

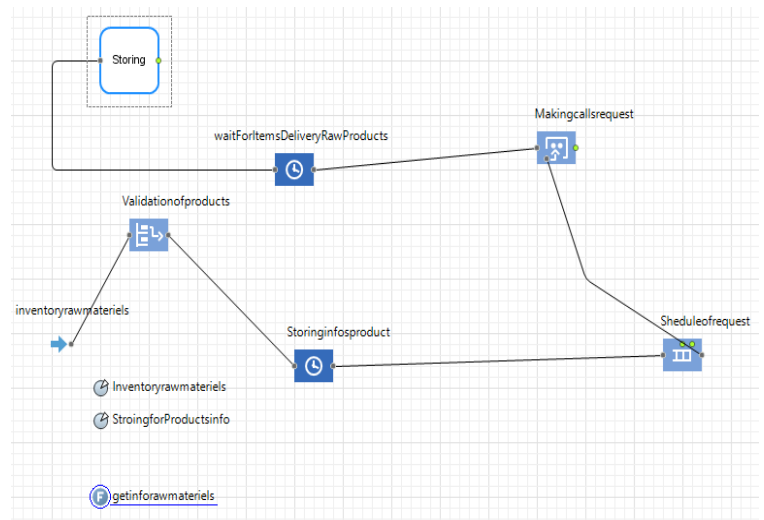

Fig. 11. Model Suppliers Scenario.

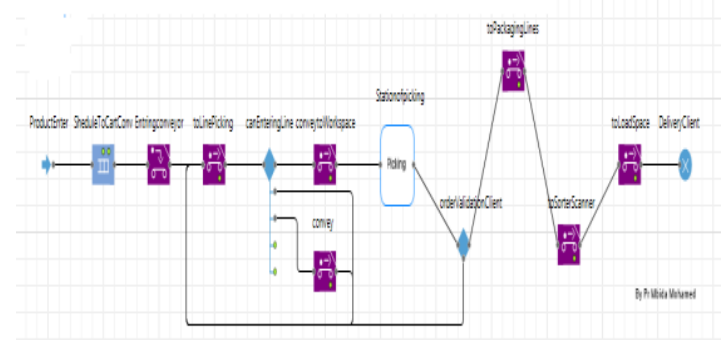

Fig. 12. CRM Model Scenario.

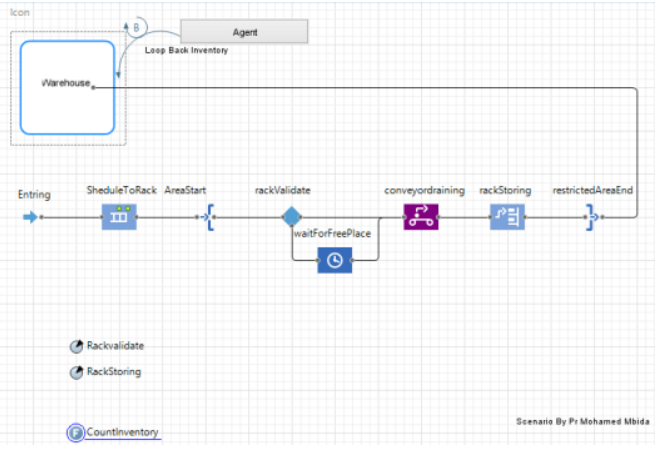

Fig. 13. Product for Delivery Scenario. 
In this part this work will simulate two cases of the Classic business model without neural network and the 2 nd with the intelligent system on the any logic platform.

NB: In the model company with NN we just keep entering and leaving the Main model with the three modules that this intelligent system constitutes the heart which manages automatically without human intervention.

\section{B. Delivery from Suppliers}

Before the start of production, each firm needs the raw material, which implies a request from the suppliers, it requires steps to follow which can generate a lot of time to exchange calls, emails ... with a risk of errors always, the model company reduces this time, by making the entire process automated in real time from the company side to the supplier. According to statistics, the simulation present that the delivery rate to the company increases in the smart model compared to the standard model which argues that the validation period with suppliers in the classic case takes a lot of time (Fig. 14, 15).

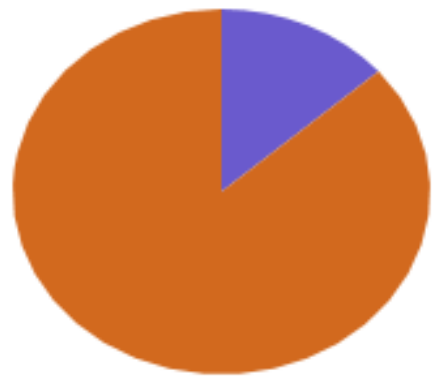

deliveny rate suppliers: $15.126(13.5 \%)$

non-deliveny rate suppliers: $96.51(86.5 \%)$

Fig. 14. Standard Delivery State of Suppliers.

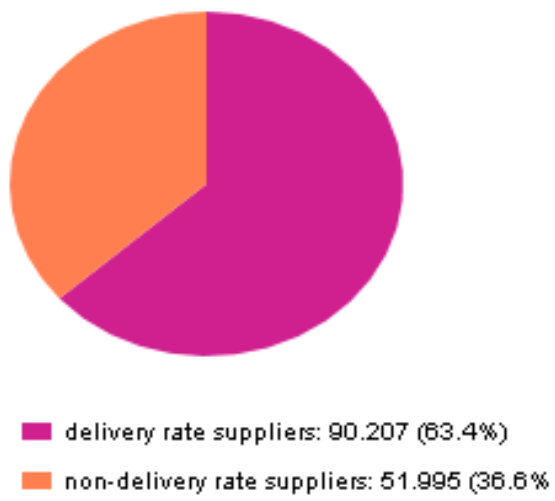

Fig. 15. Smart Delivery State of Suppliers.

\section{State of Production}

After validation of the products for manufacture with the customer, the demand enters a production queue, however this work run both simulation models to visualize the efficiency of the model with intelligent system. Each manufacturing process is characterized by precise planning by product, which comes first according to the degree of priority, in the classic model that most uses, we see that the products in manufacturing as well as the production line is at risk of overcapacity, it induces a huge waiting time of products in the state ready for production, or a cancellation of the order if the time become more longer (Fig. 16, Formula 1).

In the case of introducing the intelligent system into the business model, the queue is less congested, with a rapid execution of product orders, this is due to the elimination of validation times with the customer and the time ready for internal manufacture to the company since the intelligent system made a redefinition of the refined choices with the customers and which conforms to the standards of production, which does not require an internal validation (Fig. 17, Formula 2).

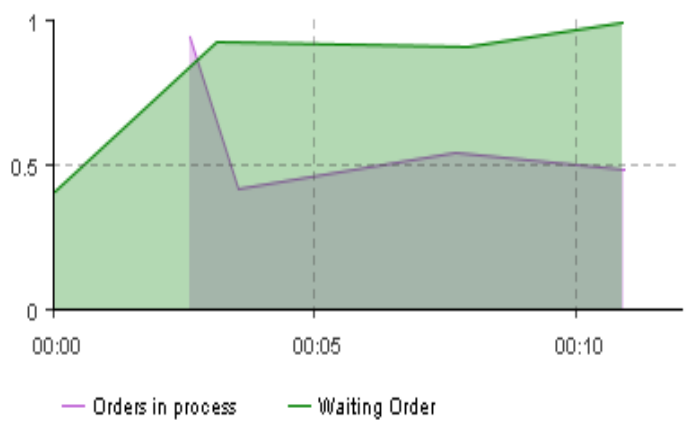

Fig. 16. Waiting Product State in Process.

Formula 1: Production start time in Standard company model

$M S=T Q+C V T+I C V T$

Indication's:

MS: Manufacturing start time (in progress)

TQ: Time in the queue

CVT: Customer validation time

ICVT: internal company validation time

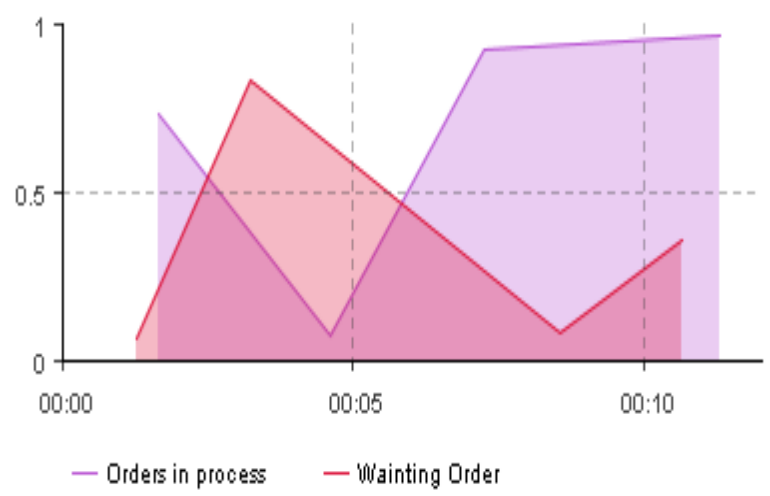

Fig. 17. Waiting Product State in Process with NN.

Nb: $\mathrm{X}$ axis: Duration of simulation in second

$Y$ axis production standby and execution 
Formula 2: Production start time in Smart company

model

$M S=C V T N N+T Q$

\section{Indication's:}

MS: Manufacturing start time (in progress)

CVTNN: Customer validation time NN

TQ: Time in the queue

\section{Time Waiting for Final Product Client}

Each customer needs between his order and the reception of these requested products a definite time between telephone calls, meetings for validation of the price, quantity ...), however with the presence of the neural network the system studies the character of the customer and validates with flexibility these choices of products as previously explained. In the business model a comparative study are made of the Customer waiting time (until obtaining his order) between the classic business model and that with the neural network (Fig. 18), the statistics related to the model on the any logic platform presented as follows:

\section{Orders}

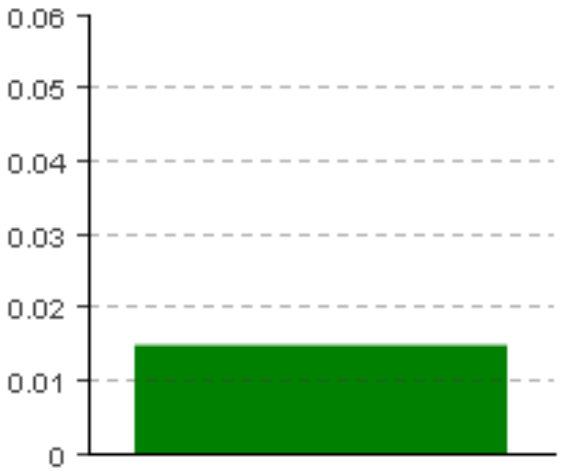

wait for deliveny product with NN: 0.01

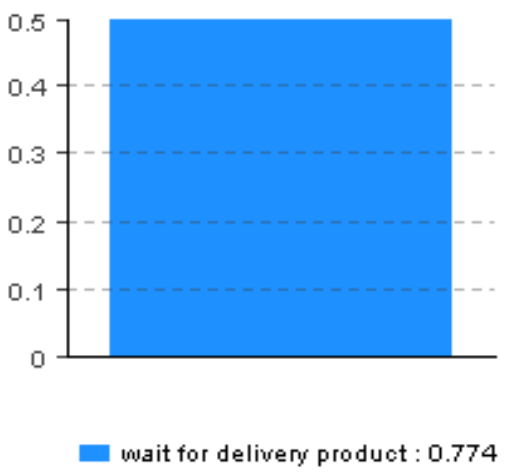

Fig. 18. Time of Waiting Client for Final Product.

$\mathrm{Nb}$ : The unit of measurement is the second
One of the customer's key preferences is to have their delivery on time, which is with a low probability in the case of a company with a standard system, as a solution to this problem has been designed the smart company model which reduces periods of time. Time for product validation in various phases and also reduced human resources by automating validation procedures (Formula 3, 4).

(Formula 3): Standard Company model

$T R F D=C V T+I V T+T Q+P T+V T B D$

Indication's:

TRFD: Time for receiving the final delivery

CVT: Customer validation time

IVT: Internal validation time

TQ: Time in the queue

PT: Production time

VTBD: Validation time before delivery

(Formula 4): Intelligent Company model

$T R F D n n=C V T n n+T Q n n+P T n n$

Indication's:

TRFDnn: Time for receiving the final delivery

CVTnn: Customer validation time of $\mathrm{NN}$

TQnn: Time in the queue

PTnn: Production time

\section{CONCLUSION}

Currently, organizations are gradually forced to manage their chains in a flexible and productive way by organizing and continuously administering in real time, to face the strong competition in the market, this leads them to automate their systems with new technologies, such as the intelligent system designed in this work, which serves to make the processing and transactions of the three basic business modules in real time with less human resources and error rates.

The perspective as a result of this work, will focus on the addition of a voice and facial recognition system in the business model by the eigenface and voice algorithm [10, 11], for a more secure access, and also it will integrate a fourth module concerning the personnel to record the hours, salaries and monthly bonus for each individual following a clocking system, this will allow instant up-to-date access to this personnel information, and will allow for an additional gain in productivity.

\section{REFERENCES}

[1] Y. Yang, *C. Li, "Doppler Radar Motion Sensor With CMOS Digital DC-Tuning VGA and Inverter-Based Sigma-Delta Modulator," IEEE Trans. Instrum. Meas., vol. 63, no. 11, pp. 2666-2674, Nov. 2014.

[2] K. Gharehbaghi*, "Artificial Neural Network for Transportation Infrastructure Systems,” MATEC Web Conf., vol. 81, p. 05001, 2016.

[3] Y. Zhang, Y.-S. Lin, I.-C. Lin, and C.-J. Chang, "Predicting logistics delivery demand with deep neural networks," in 2018 7th International Conference on Industrial Technology and Management (ICITM), Oxford, United Kingdom, 2018, pp. 294-297.

[4] F.-M. Tsai and L. J. W. Huang, "Using artificial neural networks to predict container flows between the major ports of Asia," Int. J. Prod. Res., vol. 55, no. 17, pp. 5001-5010, Sep. 2017. 
[5] N. Karna, I. Supriana and U. Maulidevi, "Social CRM using web mining," 2014 International Conference on Information Technology Systems and Innovation (ICITSI), Bandung, 2014, pp. 264-268, doi: 10.1109/ICITSI.2014.7048275.

[6] N. Guo, , Z. Wang, and J. Zhu, "GBVS Based 1D and 2D Barcodes Localization in Complex Scene," in 2015 International Conference on Computational Intelligence and Communication Networks (CICN), Jabalpur, India, 2015, pp. 352-356.

[7] MBIDA, Mohamed. Smart Warehouse Management using Hybrid Architecture of Neural Network with Barcode Reader 1D/2D Vision Technology. International Journal of Intelligent Systems and Applications, 2019, vol. 11, no 11, p. 16.

[8] O. Yorulmaz, E. Akhan, D. Tuncel, R. C. Atalay, and A. E. Cetin, "Multi-resolution super-pixels and their applications on fluorescent mesenchymal stem cells images using 1-D SIFT merging," in 2015
IEEE International Conference on Image Processing (ICIP), Quebec City, QC, Canada, 2015, pp. 2495-2499.

[9] R. Thakur and L. Workman, "Customer portfolio management (CPM) for improved customer relationship management (CRM): Are your customers platinum, gold, silver, or bronze?," J. Bus. Res., vol. 69, no. 10, pp. 4095-4102, Oct. 2016.

[10] R. Rosnelly, M. S. Simanjuntak, A. Clinton Sitepu, M. Azhari, S. Kosasi and Husen, "Face Recognition Using Eigenface Algorithm on Laptop Camera," 2020 8th International Conference on Cyber and IT Service Management (CITSM), Pangkal, Indonesia, 2020, pp. 1-4, doi: 10.1109/CITSM50537.2020.9268907.

[11] X. Zhang, Z. Tao, H. Zhao and T. Xu, "Pathological voice recognition by deep neural network," 2017 4th International Conference on Systems and Informatics (ICSAI), Hangzhou, China, 2017, pp. 464-468, doi: 10.1109/ICSAI.2017.8248337. 